\title{
Gradhiva
}

GRADHIV

Revue d'anthropologie et d'histoire des arts

29 | 2019

Estrangemental

\section{Divergence technologique. Chamanisme et numérisation en Amazonie}

Technological Divergence. Shamanism and Digitalization in the Amazon

\section{Pedro de Niemeyer Cesarino}

Traducteur : Emmanuel de Vienne

\section{(2) OpenEdition \\ Journals}

\section{Édition électronique}

URL : http://journals.openedition.org/gradhiva/4150

DOI : 10.4000/gradhiva.4150

ISSN : 1760-849X

\section{Éditeur}

Musée du quai Branly Jacques Chirac

\section{Édition imprimée}

Date de publication : 29 mai 2019

Pagination : 162-187

ISBN : 978-2-35744-112-5

ISSN : 0764-8928

\section{Référence électronique}

Pedro de Niemeyer Cesarino, «Divergence technologique. Chamanisme et numérisation en Amazonie », Gradhiva [En ligne], 29 | 2019, mis en ligne le 31 mars 2021, consulté le 02 avril 2021. URL : http://journals.openedition.org/gradhiva/4150; DOI : https://doi.org/10.4000/gradhiva.4150 


\section{Divergence technologique}

Chamanisme et numérisation en Amazonie

par Pedro de Niemeyer Cesarino

Cet article propose de réfléchir à différentes conceptions du savoir dans leur lien avec certains outils technologiques. À partir d'un cas précis - un atelier sur la numérisation de traditions orales que j'animai pour des professeurs et des chamanes marubo en Amazonie occidentale - sont ici explorées les divergences entre deux régimes de connaissance: l'un inscrit dans le corps et les connexions virtuelles qu'autorise le chamanisme, l'autre tourné vers la constitution d'archives numériques. 
1. Les Marubo sont un groupe de langue pano habitant la terre indigène du Vale do Javari (Amazonas, Brésil) et les villes voisines de Cruzeiro do Sul (Acre) et Atalaia do Norte (Amazonas). Je travaille avec eux depuis 2004.

2. L'atelier a été réalisé dans le cadre du projet «Effets de la protection des droits intellectuels et culturels sur les peuples et les savoirs traditionnels", coordonné par Manuela Carneiro da Cunha et financé par la Fondation Ford. Les résultats du projet sont rassemblés dans Cesarino et Carneiro da Cunha 2014.

3. Grande maison collective amazonienne (NdT).

4. "Double» traduit ici deux termes synonymes en marubo (vaká e yochin). Les doubles ont des qualités et des comportements corporels (ils meurent, établissent des liens de parenté, s'alimentent, etc.) et ne peuvent donc être assimilés à des "âmes ", moins encore à des représentations mentales. De manière plus large, les termes yochin et vaká signalent une ontologie de la duplication en indiquant que toute extension corporelle doit nécessairement être accompagnée de son "double" correspondant (son yochin et/ou son vaká). "Esprit », quant à lui, traduit dans ce texte le terme yove (yovevo au pluriel), agent hyper-humain qui a toujours existé. Certains " esprits" sont associés à des corps (d'oiseaux ou d'animaux par exemple); d'autres existent de manière autonome, même s'ils peuvent à l'occasion visiter les corps-maisons des chamans. Quand un esprit yove s'associe à un corps (décrit par une construction possessive, "son corps", awen shaká) ou à un animal (awen yoini), il sera considéré comme "son double (awen vakál yochin), une instanciation du lien ontologique de duplication. L'esprit de l'anaconda (vencha) pourra par exemple être désigné comme yove vencha, ou, en fonction de son association avec tel animal ou tel corps, comme yochin (en tant que double de quelque chose).

"Esprit » peut aussi désigner un « degré de magnification " de certains aspects de la personne lorsqu'ils se rapprochent de ou atteignent la qualité magnifique de ces êtres originaires. Des doubles associés au corps d'un chaman puissant sont ainsi des «quasi-esprits". Plutôt que des êtres répartis en catégories classificatoires séparées, yochin et yove désignent un mode de relation (la duplication) et yove également un mode de magnification. Pour plus de précisions sur les traductions de ces termes, voir Cesarino 2011a.

\section{Introduction}

Cet article porte sur une tentative de collaboration au succès très partiel entre des régimes de savoir qui envisagent différemment le rôle du corps ainsi que la numérisation et la virtualisation de la tradition orale. II part d'une rencontre que les Marubo ${ }^{1}$ et moi-même avions organisée dans le sillage d'une recherche antérieure sur la traduction et la documentation des arts verbaux. L'essai, cependant, devait révéler une nouvelle facette de l'incommensurabilité qui caractérise les relations entre les chamanismes amazoniens et les modalités académiques de circulation et de production du savoir. Cette incommensurabilité indique, mieux encore, que les technologies corporelles amérindiennes sont ouvertes au virtuel d'une manière telle qu'elles rendent partiellement inutiles les monuments de documentation numérique et graphique que génèrent nos protocoles de recherche. Le cas en apparence singulier et banal décrit dans cet article montrera aussi des détails sur ce qu'on a coutume d'appeler les «ornements " corporels amérindiens, lesquels gagneraient à être qualifiés, en l'occurrence, de dispositifs de connexion. En 2012, à Cruzeiro do Sul (dans l'État de l'Acre, au Brésil), au moment d'animer cet atelier de documentation à destination des Marubo, j'ai eu la surprise de découvrir que mes amis utilisaient des ornements en perles que je n'avais jamais vus avant. L'atelier avait été organisé à la demande des Marubo comme une contrepartie supplémentaire à la grande recherche que j'avais réalisée les années précédentes dans leurs communautés ${ }^{2}$. L'objectif plus spécifique était d'opérer un «transfert technologique» en formant une équipe de Marubo à l'enregistrement numérique de performances orales, à la production de métadonnées, à l'organisation d'informations et d'annotations, afin de constituer une grande banque de données sur leur riche tradition des arts de la parole hébergée dans un centre sur leur territoire et au Museu do Índio à Rio de Janeiro. Pour ce faire, je devais passer cinq jours dans un hôtel avec un groupe d'une dizaine de Marubo que je connaissais depuis longtemps. Certains des participants étaient donc arrivés parés de bracelets et de bandeaux au répertoire iconographique inédit. Plus préoccupé de conduire l'atelier que d'ethnographie, je n'y vis d'abord rien d'important et ne cherchai pas à en savoir davantage. Les jours suivants, Reinaldo Venãpa, un des professeurs marubo, surpris de mon manque de curiosité, entreprit de m'expliquer le sens de ces ornements. Ce n'est cependant qu'en commençant à écrire ces lignes que j'ai mesuré la portée de ses paroles et pris conscience de leur rapport avec la situation où nous nous trouvions. Tout se passait comme si était en jeu une sorte de médiation esthétique et technologique entre deux épistémologies: celle que je représentais et celle des Marubo.

Pour déployer ces pistes de réflexion, quelques remarques introductives sur des thèmes que j'ai abordés dans des travaux antérieurs sont nécessaires (voir en particulier Cesarino 2011a). La première concerne la personne marubo, et en particulier celle de Robson Doles Venãpa, un chaman romeya, chercheur indien et instituteur dans sa communauté (Cesarino 2014). La seconde porte sur les autres répertoires ornementaux et motifs graphiques des Marubo, qui éclaireront par contraste les innovations de 2012. La personne marubo est constituée d'une famille de doubles qui se pensent comme des frères et qui, résidant de notre point de vue à l'intérieur du corps, se voient comme habitant une maloca ${ }^{3}$. Ceux que l'on peut qualifier de «vivants " (kayakavi, ce qui a l'aspect d'un corps, kaya) sont constitués d'une enveloppe ou plus exactement d'une carcasse qui abrite divers doubles ${ }^{4}$ 
(vakárasĩ), réplication exacte de la maloca extérieure où vivent les Marubo. Les trois principaux doubles (du cœur, du côté droit et du côté gauche) sont ordonnés selon le rang de naissance par puissance - intellectuelle et chamanique - décroissante. Le double du cœur, le plus vieux du trio (chinã nató ${ }^{\mathbf{5}}$ ), qui est «presque comme un esprit», est ainsi de fait considéré comme un chaman. Grâce à ses capacités extraordinaires, bien supérieures à celles du corps-enveloppe, il peut sortir sans peine de sa maison. II arpente le cosmos, visite une foule d'autres esprits qui le peuple et retourne à sa maison-corps, riche de savoirs nouveaux. En son absence, quand la maison est vide, il peut inviter d'autres esprits ou d'autres doubles (de morts, par exemple) pour y chanter: c'est là une capacité exclusive des chamans romeya. Dans cette maloca "visible» (c'est-à-dire le corps du chaman), les autres chamans maîtres des incantations kẽchĩtxo écoutent ces chants et ces enseignements venus d'ailleurs et enclenchent ainsi le processus de transmission des savoirs ${ }^{6}$. Robson, comme potentiellement tous les chamans et les autres Marubo, est une personne complexe, divisée entre plusieurs parties du corps dotées chacune de son double:

\begin{tabular}{|c|c|c|}
\hline Nom & Classification & État \\
\hline $\begin{array}{l}\text { Robson Doles } \\
\text { Venãpa }\end{array}$ & $\begin{array}{l}\text { kaya } \\
\text { (extension corporelle) } \\
\text { shaká (carcasse) } \\
\text { yora (corps/personne) }\end{array}$ & $\begin{array}{l}\text { mashteya } \\
\text { (mûr, complet) }\end{array}$ \\
\hline Isko Osho & $\begin{array}{l}\text { chinã nató } \\
\text { (double du cœur, } \\
\text { frère aîné) }\end{array}$ & mashteya \\
\hline Panã & $\begin{array}{l}\text { mekiri vaká } \\
\text { (double du côté droit, } \\
\text { frère cadet) }\end{array}$ & mashteya \\
\hline Pei & $\begin{array}{l}\text { mechmiri vaká } \\
\text { (double du côté } \\
\text { gauche, benjamin) }\end{array}$ & mashteya \\
\hline Vimini & $\begin{array}{l}\text { txipokeshta } \\
\text { (frère cadet) }\end{array}$ & $\begin{array}{l}\text { était un enfant } \\
\text { en } 2007 \text {, a grandi } \\
\text { et est désormais } \\
\text { mashteya }\end{array}$ \\
\hline
\end{tabular}

Tableau 1. La composition de la personne Robson Venãpa.

La famille virtuelle qui caractérise la personne multiple n'est pas figée. Elle peut se modifier comme les nôtres. C'est à partir d'une réflexion sur cette composition de la personne que Reinaldo Venãpa a commencé à m'expliquer le sens des ornements. Tous ces doubles voyagent et rapportent des
5. Chinã nató signifie littéralement «noyau de la poitrine-pensée».

6. C'est la différence essentielle entre les deux types de chamanisme existant chez les Marubo: la capacité d'externaliser les doubles et d'en recevoir d'autres à l'intérieur de soi (caractéristique des romeya) versus l'action par les chants et les esprits auxiliaires (une prérogative des kẽchĩtxo). 


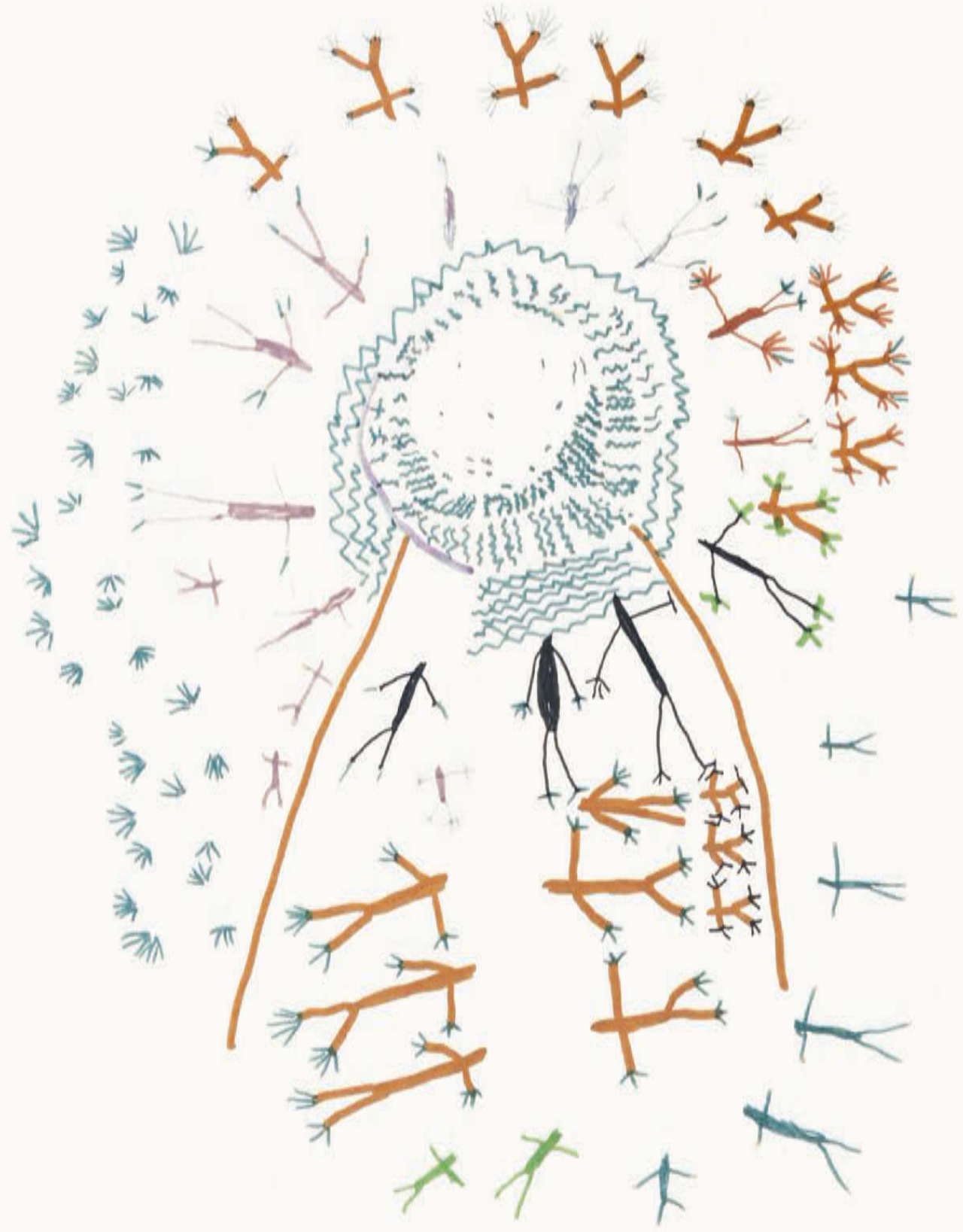




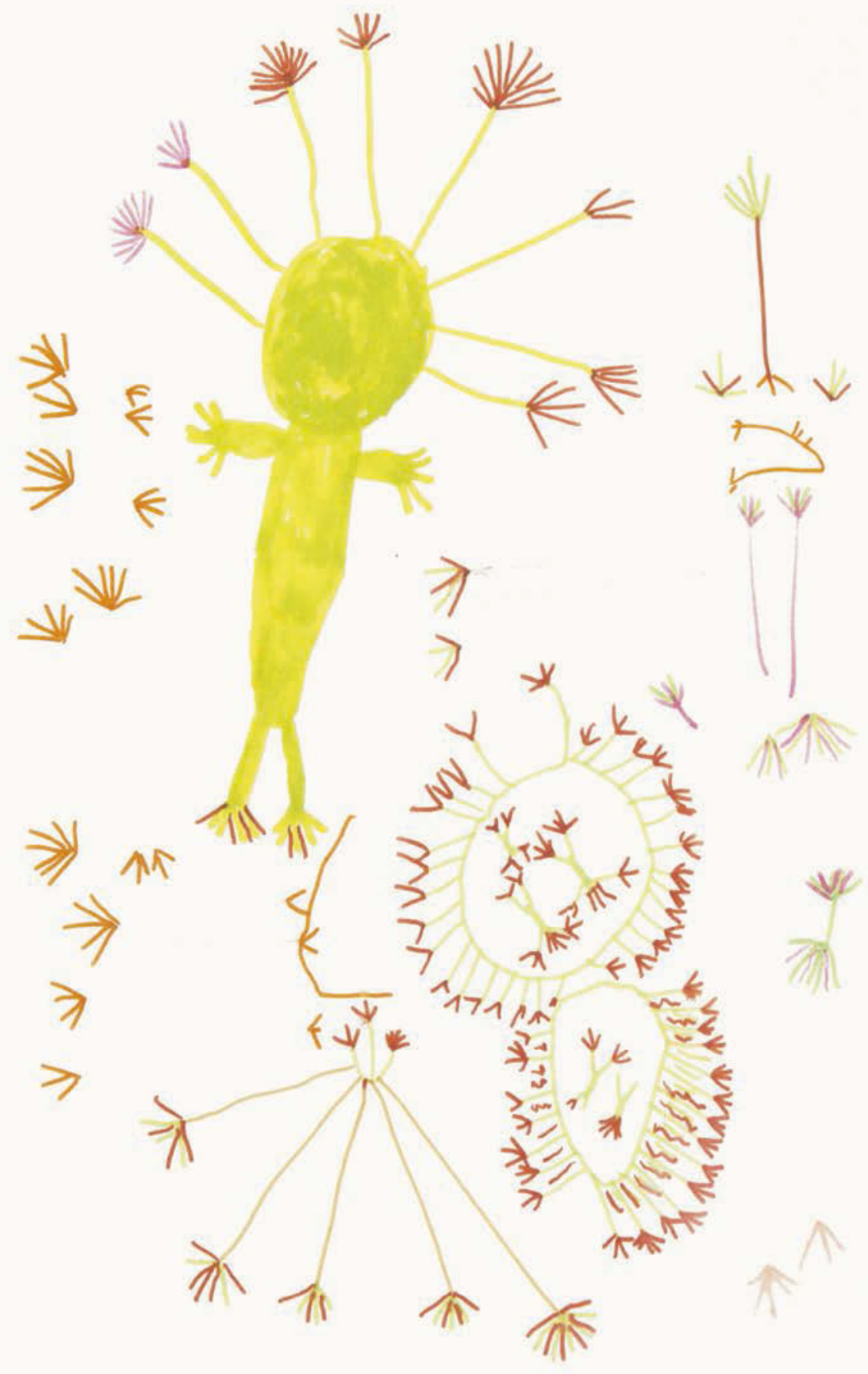


7. Les parents alliés à Robson Venãpa proviennent de diverses communautés du haut Rio Ituí (terre indigène Vale do Javari, Amazonas, Brésil), en particulier Alegria, Paraná et Vida Nova. Ce groupe est en rivalité (sans hostilité) avec les habitants du Rio Curuçá, où se trouvent les plus grandes et les plus anciennes maloca marubo. Pour des raisons que j'ignore, cela fait environ quarante ans que le Rio Ituí regroupe tous les chamans romeya actifs, en plus de très nombreux incantateurs kenchintxo. Même s'il n'existe pas de romeya sur le Curuçá, les incantateurs qui y officient sont unanimement considérés comme les meilleurs spécialistes des arts verbaux et des autres savoirs rituels marubo Dans ce contexte, Robson Venãpa semble occuper une place particulière. Sans doute pour avoir été un des premiers traducteurs de la New Tribes Mission, puis dans la foulée mon professeur et principal interlocuteur pendant ma recherche (voir Cesarino 2014 pour davantage d'informations biographiques), Robson est devenu un médiateur habile avec les Blancs intellectuels. Mais il est par-dessus tout un chaman puissant grâce à sa capacité à se lier avec des doubles et des esprits qui ne sont pas toujours accessibles aux autres romeya (Armando et José Nascimento étaient les deux autres romeya en activité au moment de ma recherche). Cette connexion est souvent décrite par Robson comme une source d'innovation dans sa manière de transmettre le savoir et de former d'autres chamans incantateurs (en plus d'être une source de savoir encyclopédique qu'il transmet à ses parents) : par leurs voyages continuels, ses doubles (surtout Isko Osho) apprennent auprès d'autres chamans virtuels et finissent par transformer les rituels, savoirs et ornements corporels, comme ceux qui sont discutés ici.

\section{Pomacea canaliculata.}

\section{double page précédente}

fig. 1

À gauche: Orlando Nakeuxima, Motoka et ses fils, dessin yanomami, s.d. Collection Laymert Garcia dos Santos et Stella Senra.

fig. 2

À droite: Orlando Nakeuxima, La maison des esprits, dessin yanomami, s.d. Collection Laymert Garcia dos Santos et Stella Senra. connaissances des quatre coins du monde. Ils restent peu dans la maisoncorps de Venãpa. Par exemple Isko Osho, le frère aîné, a pour habitude de conduire les doubles des morts égarés et voyage un peu partout: Robson Venãpa m'a raconté qu'il s'est trouvé une fois au Bangladesh pour guider les morts d'un tremblement de terre. On voit ainsi que «Robson » est devenu une sorte de lieu de réunion et de dispersion de ces agents multiples qui peuplent le cosmos et forment un régime complexe de connaissance virtuelle. II est devenu en outre l'annonciateur d'une apocalypse imminente qui surviendra lorsque Kana Voã, le démiurge de la cosmologie marubo, reviendra de sa demeure. Un grand incendie couvrira alors la terre entière et Kana Voã séparera les personnes qui vivent selon ses préceptes de celles qui vivent selon les mauvais. Robson (à travers ses doubles) a également réuni et dirigé un groupe de personnes appartenant à différents segments de la société des Marubo du Haut Ituí (Isko Nawavo, Shane Nawavo, Shawã Nawavo, Rovo Nawavo e Vari Nawavo). Ce groupe est composé de certains de ses parents qui se sont soumis à un intense entraînement chamanique, dont j'ai accompagné quelques étapes. Un des buts de cet entraînement est de parvenir à retenir ces enseignements dans leurs propres corps afin qu'ils ne se dispersent ou ne s'oublient pas au contact des odeurs et des fluides de ce monde. Le groupe de Robson, ainsi, «vit uni pour penser » (chinãyai shokosho) et non "pour faire la guerre/se disputer» (pakayai shokosho), comme, aiment-ils à dire, les chercheurs et les missionnaires non Indiens ${ }^{7}$.

\section{L'ornementation corporelle marubo}

En règle générale, les ornements marubo ne sont pas porteurs d'un sens spécifique. Ils indiquent tout au plus une relation indirecte avec le chamanisme. Ceux qui sont faits en coquille d'escargot-pomme ${ }^{8}$ (novo) sont réputés détenir une chinã (force, principe vital) et protéger les chamans qui les portent des attaques d'esprits yovevo grâce à leur clarté et leur éclat. On dit aussi qu'ils sont «l'or des Indiens » par leur rareté et la difficulté de leur fabrication. En outre, ils aident le double des défunts dans leur parcours sur le «chemin des morts». Ils rendent enfin la personne «bonne/belle/correcte» (roaka), autrement dit "prototypique" (yora koin), par opposition aux étrangers (nawa) et aux ennemis (rawin). Sur les autres ornements en perles qu'ils produisent, les Marubo, à ma connaissance, disent peu de choses. Une partie de la production récente semble inspirée par celle d'autres groupes panos voisins, comme les Huni Kuin et les Yawanawa. Les chamans portent des bandoulières en perles bleu foncé; les femmes une profusion de grosses ceintures, de colliers et de bandoulières en perles de couleur. Aucun de ces ornements ne suscite d'exégèses approfondies: ils sont destinés à "faire joli ». Quelques rares pièces, cependant, montrent des dessins réservés aux chamans (confirmés ou néophytes), comme celles élaborées à partir des motifs shonõ shena, veken kene et tao peika. Les jeunes et les enfants doivent les éviter. Le motif tao peika est aussi appelé venchan kene, motif anaconda, et vei kene, motif mort. Des précautions entourent aussi l'usage des motifs paka mevi kene, en peinture corporelle ou sur les ornements. Ce dessin guerrier peut engendrer un comportement agressif chez celui qui le porte. Cet ensemble d'ornements renvoie au problème plus large de l'inscription des motifs graphiques dans la construction de la personne, que j'ai abordé ailleurs (Cesarino 2011b, 2012). Retenons-en que ces motifs chamaniques 
peuvent être employés, sous forme de peinture corporelle, à l'occasion des longs entraînements au chamanisme ou des rituels où sont présents les esprits. L'application de ces peintures rythme l'acquisition graduelle d'une "maturité» (mashteya) de la force-pensée (chinã). Elles ne pourraient par conséquent être utilisées par quelqu'un, par exemple un adolescent, qui n'aurait aucune familiarité avec le savoir (ese). Les doubles de Robson ont atteint cet état de maturité, comme le montre le tableau p.165. Ils ont désormais été intégralement recouverts de peintures par leurs «tantes-esprits».

Chez les Marubo comme chez de nombreux autres Amérindiens, on dessine sur son corps pour imiter celui des esprits, jugé plus beau, plus parfumé, plus grand et plus décoré. Par exemple, porter les motifs shonõ shena et veken kene permet d'être identifié comme une personne (comme un parent en puissance) par les esprits yovevo, un aspect crucial des rituels d'initiation où le néophyte doit rêver d'un parent indéfini qui lui offrira certains objets (comme des radios CB ou des aras), porteurs de son chinã et de sa capacité de parole et de mémoire. La beauté est donc l'ingrédient indispensable à cette reconnaissance onirique par un agent puissant.

Néanmoins, l'étude des noms ou des formes des motifs élémentaires ne permet pas d'en déduire des significations particulières. Leurs noms, comme "larve de samaúma», n'impliquent pas que les motifs soient des représentations d'objets auxquels ils font référence, pas plus qu'ils n'éclairent ce qui motive ces compositions. L'onomastique semble relever, comme l'a suggéré Pierre Déléage (2007), d'une technique qui facilite sa mémorisation en mobilisant une saillance visuelle dans l'environnement. Plus révélateur est l'autre nom du motif, sa macro-catégorie: yove kene. Signifiant littéralement "motif esprit», il désigne un dessin qui construit ou transforme la personne, la rapprochant de la qualité d'esprit. Enfin, de très nombreux motifs inscrits sur le corps d'une personne et sur celui de ses doubles sont fréquemment comparés aux passeports des Blancs ainsi qu'à l'exercice de la traduction. Chaque collectif du cosmos a ses propres motifs qui permettent d'en identifier les membres. Ceux de mon double, à titre d'exemple, sont différents de ceux des yora koin, les «personnes prototypiques", car il vit avec des esprits étrangers au Rio Sol (Vari Waka). Les Marubo m'ont appris que c'est grâce à ces doubles inscrits sur son propre corps que mon frère-double aîné, Varin Pena, a pu acquérir la maturité nécessaire pour pouvoir m'aider à apprendre la langue marubo (Cesarino 2011a, 2012).

Ces liens entre une partie de la production esthétique marubo, la production de la personne et le chamanisme n'expliquent cependant qu'imparfaitement la nouvelle collection de bracelets, de brassards et de bandeaux qu'exhibaient les participants à l'atelier de 2012. J'avais jusqu'alors écarté la pertinence d'une recherche de significations, d'un décodage textuel ou symbolique des compositions graphiques ou de la culture matérielle. De fait, j'avais volontairement abandonné l'ensemble des questions posées à l'anthropologie de l'art depuis Primitive Art de Franz Boas, puis relancées par la sémiologie et la linguistique structurale. Les présupposés sémiotiques de ces approches (la quête du symbolisme, la recherche d'unités visuelles minimales qui se comporteraient de manière analogue aux phonèmes, la recherche de possibles relations de sens, etc.) ont été critiqués à partir des années 1980 par les tenants du «tournant iconique» (pictorial turn), qu'ils fussent théoriciens de l'image et de l'art, comme W.J.T. Mitchell, ou anthropologues, comme Alfred Gell. La recherche de sens ou de symboles cachés dans les 
9. L'expression awen take (possessif frère/paire) désigne en marubo un compagnon, une paire de personnes ou d'autres phénomènes qui sont incompréhensibles en tant qu'unité. Le terme take veut dire "frère" (une relation de germanité dans le système de parenté), mais aussi "paire», au sens arithmétique.

10. Pour plus d'informations sur l'organisation sociale, le système de parenté et la logique de classification marubo, voir : Melatti 1977 ; Cesarino 2018 Le néologisme

"sociocosmique" reconnaît le fait que les liens de parenté débordent le socius humain (du point de vue moderne) et valent pour les esprits et les doubles d'animaux, situés à d'autres positions cosmologiques. répertoires graphiques (comme si toute production visuelle ou graphique était en dernière instance un texte de la culture ou de la cosmologie d'un groupe donné), assez présente dans l'ethnologie des basses terres d'Amérique du Sud jusqu'aux années 1990, a été à son tour remise en cause par des auteurs comme Aristóteles Barcelos Neto (2008), Peter Gow, (1999), AnneChristine Taylor (2003) et d'autres. Cela ne veut pas dire que les relations entre image et langage n'ont aucune pertinence pour comprendre les régimes visuels amérindiens. Si le modèle sémiologique ne suffit pas à saisir des traditions comme celles des dessins kene, il reste à considérer un ensemble d'autres répertoires qui sont précisément étroitement articulés à des discours. C'est le cas des iconographies pictographiques que Déléage (2007, 2017), Severi (2007) ou moi-même $(2012,2015)$ avons étudiées. Elles révèlent des affinités profondes avec d'autres écritures pictographiques amérindiennes consolidées - celles entre autres des Kuna, des Ojibwa ou des Nahuatl de l'ancien Mexique -, qui justifient qu'on essaie de saisir la signification des images.

\section{Duplication et grammaire visuelle}

Les ornements utilisés par mes amis lors de cet atelier étaient ainsi sui generis. Alors que les pictographies amazoniennes sont nées de la relation avec des non-Indiens, comme des anthropologues, responsables de la création d'une nouvelle forme d'expression à même de communiquer certaines informations narratives, les ornements de l'atelier étaient pour leur part une invention spontanée. D'un côté, ils ressemblent aux ornements produits par leurs voisins Huni Kuin et Yawanawa qui alimentent de plus en plus les boutiques d'art indien, de l'autre, leur composition graphique est assez singulière et totalement absente des circuits de commercialisation. Comment comprendre le surgissement soudain de ce qui mérite d'être qualifié de grammaire visuelle? L'existence d'une grammaire ne dispense pas d'envisager celle-ci à partir de son usage, sa circulation et son activation rituelle. Une iconographie ne saurait être conçue de manière abstraite, à la manière des registres ethnographiques des «pièces » et des «patterns » de la culture matérielle, invariablement décollés de leurs contextes d'énonciation et de circulation lorsqu'ils se transforment en illustrations imprimées ou en objet du regard artistique, didactique ou scientifique.

Commençons par la signification des couleurs. Reinaldo, un des jeunes professeurs participant à l'atelier, m'a expliqué qu'elles renvoient aux esprits auxiliaires et aux doubles de Robson Venãpa. Le tableau suivant complète le précédent en associant des paires de couleurs aux paires de doubles et d'esprits yovevo (Isko Osho forme une paire avec Wiren, et ainsi de suite). Sur la première ligne, deux doubles de Robson forment une paire (awen take, comme l'exprime la langue marubo ${ }^{9}$ ); à la ligne suivante, un de ses doubles est apparié à l'esprit Kana Panã. Dans les autres, les paires sont constituées seulement d'esprits. On voit ainsi que le réseau d'alliances de Robson, loin de se limiter à la triade des doubles associés depuis sa naissance à son corps-maloca, s'étend à d'autres alliés puissants, les yovevo. Les paires sont ordonnées à partir de la relation asymétrique de germanité (aîné/cadet) qui structure le système de parenté sociocosmique marubo ${ }^{10}$ et s'inscrivent évidemment dans le problème général de l'asymétrie dans la pensée amérindienne (Lévi-Strauss 1991). Les doubles (vaká, yochin) les plus développés, 
ou «mûrs", sont considérés par les Marubo comme des quasi-esprits (yovepase) car ils partagent presque leurs qualités extraordinaires. Les esprits proprement dits (comme Kana Panã, mentionné plus bas), quant à eux, ont une existence indépendante de la personne puisqu'ils lui préexistent et lui survivent, mais peuvent tisser avec elle une relation d'alliance.

\begin{tabular}{|c|c|c|}
\hline & Aîné (vevoke) & Cadet (txipoke) \\
\hline Double/double & Isko Osho & Wiren \\
\hline Couleur & $\begin{array}{l}\text { jaune } \\
\text { (ratãshka) }\end{array}$ & $\begin{array}{l}\text { bleu clair } \\
\text { (shane koroka) }\end{array}$ \\
\hline Esprit/double & Kana Panã & Vimini \\
\hline Couleur & $\begin{array}{l}\text { orange } \\
\text { (ranchinka) }\end{array}$ & $\begin{array}{l}\text { blanc } \\
\text { (oshoka) }\end{array}$ \\
\hline Esprit/esprit & Kapi Kene & Waka Panã \\
\hline Couleur & $\begin{array}{l}\text { vert } \\
\text { (panã shakápa) }\end{array}$ & $\begin{array}{l}\text { noir } \\
\text { (txesheka) }\end{array}$ \\
\hline Esprit/esprit & Shaká Papia & Kanã Sheta 11 \\
\hline Couleur & $\begin{array}{l}\text { bleu foncé } \\
\text { (shane isoka) }\end{array}$ & $\begin{array}{l}\text { rouge et rose } \\
\text { (onchinka, onchin } \\
\text { koroka) }\end{array}$ \\
\hline
\end{tabular}

Tableau 2. Relations entre paires de doubles ou d'esprits et couleurs.

Dans cette série, toutes les paires sont composées d'agents reliés et alliés à la personne complexe identifiée (partiellement) comme étant Robson Venãpa. La paire prééminente est composée d'Isko Osho et Wiren, car Isko Osho est le double du cœur (chinã nató) de Robson, et donc son frère aîné virtuel (otxi), accompagné de son partenaire, son cadet Wiren. Les autres paires sont importantes également et ont pour habitude de s'associer à chacun des participants du groupe rituel de Venãpa, comme nous allons le voir.

Examinons maintenant en détail la composition de la première pièce du répertoire (fig. 3), appartenant à Reinaldo Venãpa. Le jaune, sa couleur dominante (celle du fond), rattache ce brassard à Isko Osho, et indique que celui-ci a établi une relation spéciale avec Reinaldo, qu'il est son «professeur» (terme qu'emploient les Marubo) et «protecteur» (vesoya) privilégié. Les autres doubles et esprits sont néanmoins présents dans la composition à travers les autres couleurs ${ }^{\mathbf{1 2}}$. Ainsi, la première frange de perles rouges en partant de la droite (à l'arrière de la pièce) fait référence à Kanã Sheta; la deuxième, bleu foncé, à Shaká Papia; la troisième, verte, à Kapi Kene; la quatrième, noire, à Waka Panã ; la cinquième, bleu clair, à Wiren; la sixième, jaune, à Isko
11. Kanã Sheta est le seul esprit féminin de cet ensemble. Les autres sont masculins.

12. Le nom Isko Osho, bien qu'il puisse être traduit littéralement comme "cassique blanc", n'établit aucune relation entre l'oiseau cassique, la couleur blanche et le double de Robson. "C'est juste son nom" (awen anerivi), comme disent les Marubo. 

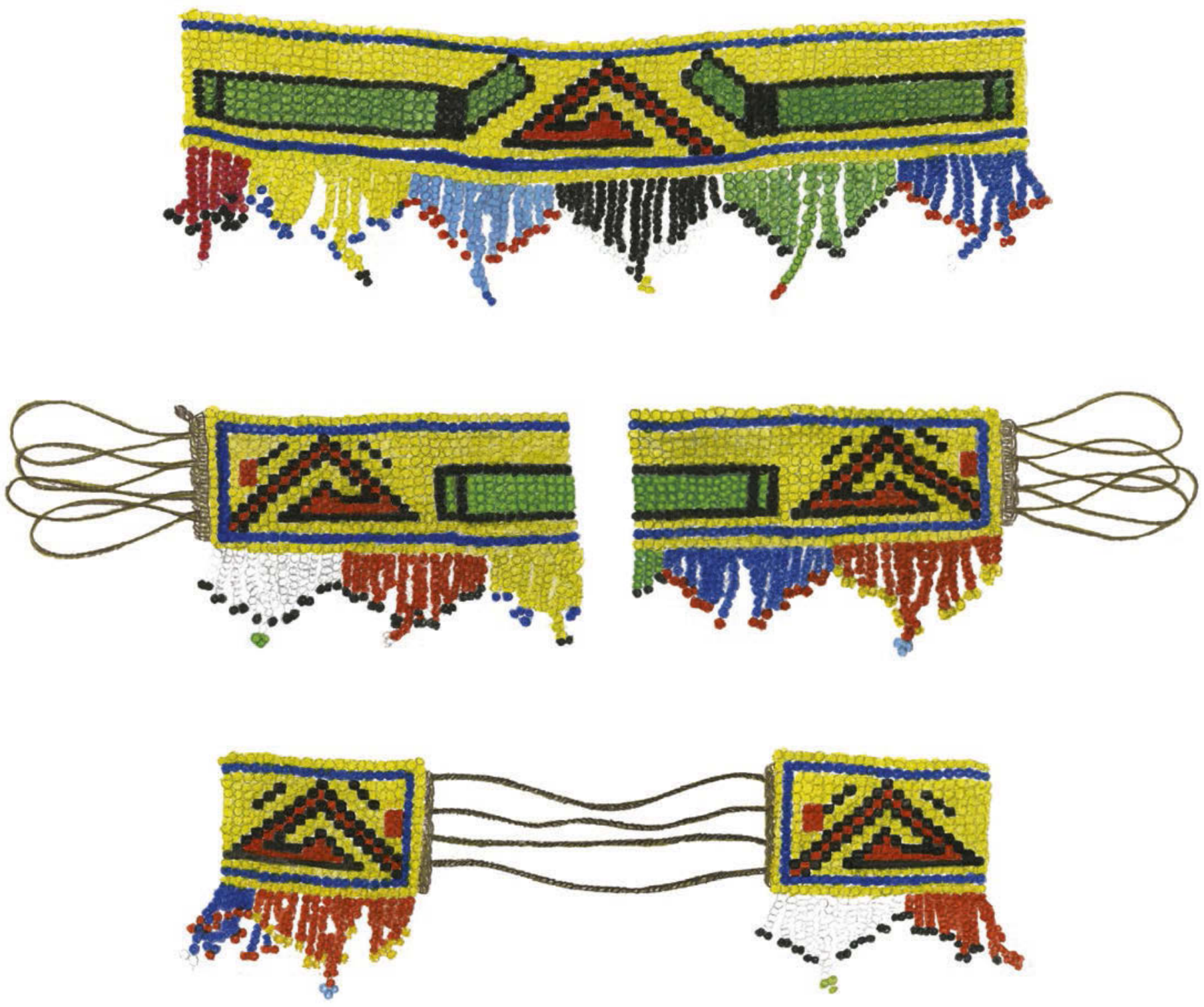
Osho; la septième, orange, à Kana Panã et enfin, la huitième, blanche, à Vimini. Sur cette pièce, les couleurs associées aux doubles sont organisées exactement comme dans le tableau 2 (de droite à gauche):

1) Kanã Sheta (rouge) - 2) Shaká Papia (bleu foncé)

3) Kapi Kene (vert) - 4) Waka Panã (noir)

5) Wiren (bleu clair) - 6) Isko Osho (jaune)

7) Kana Panã (orange) - 8) Vimini (blanc)

La série complète des brassards de Reinaldo, où prédomine Isko Osho, signale l'alliance que les membres de ce groupe de doubles ont contractée entre eux. C'est une alliance de parenté et de pensée, car les esprits et les doubles vivent ensemble, chantent et échangent des savoirs en plus de se concevoir comme des frères (take) associés à leurs segments sociocosmiques respectifs (kana et isko en l'espèce, qui sont les segments auxquels appartient la personne complexe Robson Venãpa). Cette alliance se réplique entre les vivants, car les esprits et doubles de Venãpa prennent sous leur tutelle les doubles intérieurs des membres de son cercle. Ils en sont les protecteurs (awen vesoyavo), c'est-à-dire qu'ils leur apprennent à penser et à vivre mieux grâce aux soins qu'ils dispensent à leurs doubles. Le même code de couleurs vaut également pour les motifs situés entre les deux lignes bleu foncé, sur la partie supérieure du brassard. Cet espace est défini exactement comme un intérieur, un corps (kaya) ou une maloca (shovo), c'est-àdire comme le corps-maloca de la personne marubo où résident les doubles internes. Quels sont donc ces motifs?

\begin{tabular}{|l|l|}
\hline Unité minimale & Signification \\
\hline carré & porte/pourvoyeur de soins \\
\hline triangle (motif veken) & personne/pensée \\
\hline inhalateur de tabac moulu & parole et pensée \\
\hline diamant & personne \\
\hline calebasse d'ayahuasca & personne (apprenti) \\
\hline pot d'ayahuasca & personne (apprenti) \\
\hline
\end{tabular}

Tableau 3. Les motifs élémentaires et leurs sens.

À chaque extrémité se trouvent deux carrés orange, qui sont les portes de la maloca, suivis de deux motifs veken kene (réservés aux chamans), qui sont la métaphore visuelle d'une figure protectrice ou pourvoyeuse de soins (yora vesoya) de cette maison-corps (sans avoir pu le confirmer, je suspecte qu'il s'agisse de Kana Panã, compte tenu de leur couleur orange). À côté sont disposés deux inhalateurs de tabac (rewe) stylisés, instruments importants du chamanisme marubo qui font office de soul catcher et de soul deliverer (c'est-à-dire des attracteurs et des véhicules pour les doubles et les principes

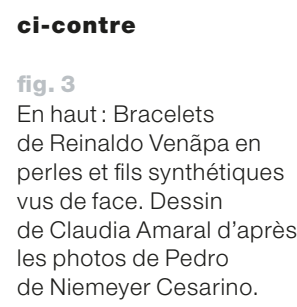

Au milieu: Vues du même bracelet côté gauche et côté droit. Dessins de Claudia Amaral d'après les photos de Pedro de Niemeyer Cesarino.

En bas: Vue du même bracelet, au verso. Dessin de Claudia Amaral d'après les photos de Pedro de Niemeyer Cesarino. 
ci-contre

fig: 4

En haut: Bracelets de Reinaldo Venãpa en perles et fils synthétiques vus de face. Dessin de Claudia Amaral d'après les photos de Pedro de Niemeyer Cesarino.

Au milieu: Vues du même bracelet côté gauche et côté droit. Dessins de Claudia Amaral d'après les photos de Pedro de Niemeyer Cesarino.

En bas: Vue du même bracelet, au verso. Dessin de Claudia Amaral d'après les photos de Pedro de Niemeyer Cesarino. vitaux), mais aussi de jumelles et de stéthoscopes pour écouter et observer les maladies et les esprits, proches ou lointains. L'inhalateur de gauche est la métaphore visuelle de la pensée (chinã) [fig.3]; celui de droite, des paroles et de l'apprentissage (yoã). Le motif triangulaire (veken kene) au milieu du brassard, enfin, est une autre métaphore de la personne, car les gens en ont le corps recouvert.

Cette série de dessins signifie que le détenteur du brassard participe aux enseignements transmis par les esprits et les doubles de Robson, auxquels elle fait référence de manière systématique. Elle indique, de manière plus précise, que le savoir ne doit pas s'échapper de la personne, de la même manière que les individus doivent rester à l'intérieur de leurs maisons, protégés par des portes. Elle dit encore que le savoir est lié de manière essentielle aux récits et aux chants (yoã vana), représentés par un des inhalateurs, et à la pensée/force vitale (chinã), représentée par le second inhalateur. En portant cet ornement, ce qu'il faut faire nuit et jour, son détenteur n'oubliera pas les savoirs et pourra parfaire sa formation. II sera en outre identifié comme un membre du groupe des «penseurs» (chinãyavo yora) dirigé par Robson Venãpa.

Cette grammaire efficace, pourrait-on dire, vaut également pour la figure 5 comme pour toutes les autres de ce répertoire. Sur ce brassard, utilisé lui aussi par Reinaldo Venãpa, la distribution des doubles et des couleurs dans la frange répond au même principe que celle du précédent. Le triangle qui entoure le motif veken kene sur le corps du brassard respecte le même code de couleurs: la ligne blanche, extérieure, renvoie à Wiren; la ligne verte, à l'intérieur, à Kapi Kene, et la ligne noire à Waka Panã. Si la couleur blanche du fond assigne cette pièce dans son ensemble à Vimini, le motif orange semble renvoyer à Isko Osho.

De nouveau, le corps de l'ornement est le corps-maloca de la personne, fermé aux deux bouts par des portes (les grands carrés aux hachures obliques), métaphores visuelles d'une personne attentive qui empêche les savoirs appris de sortir vers l'espace extérieur. Les deux motifs orange figurent la pensée (chinã) et occupent ainsi la même position que les inhalateurs sur la pièce précédente. Le diamant central est une métaphore de la personne (représentée par un triangle dans l'exemple précédent [fig.3]) qui doit recevoir et fixer le savoir (ici associé à Wiren par la couleur bleu ciel du diamant).

Le bandeau de la figure 5 est porté par Benedito Keninawa, beau-père de Robson Venãpa, apprenti chaman et le plus âgé des professeurs marubo. Une fois encore, les deux croix qui occupent les extrémités sont les gardiens qui empêchent la sortie de la personne et/ou de ses savoirs de l'espace intérieur du corps-maloca, que Venãpa compare en l'occurrence à «l'intérieur d'une église" (igreja naki keská). Les deux dessins qui suivent les croix sont des pots pour conserver l'ayahuasca (oni chomo), métaphores des «autres personnes présentes dans cet espace et qui désirent aussi apprendre " (yora wetsarasin yositsiki). L'inhalateur bleu désigne la «parole pensée» (chinã vana), soit l'ensemble de chants de cure qu'un chaman incantateur doit apprendre. Juste à côté se trouve une calebasse pour servir l'ayahuasca (oni mãsen), autre métaphore visuelle d'une personne qui souhaite apprendre. L'inhalateur orange renvoie enfin à la "parole d'enseignement», ou "parole respectueuse " (ese vana), un ensemble de préceptes éthiques et philosophiques d'ordinaire transmis à travers un art verbal spécifique. Une fois encore, l'espace 

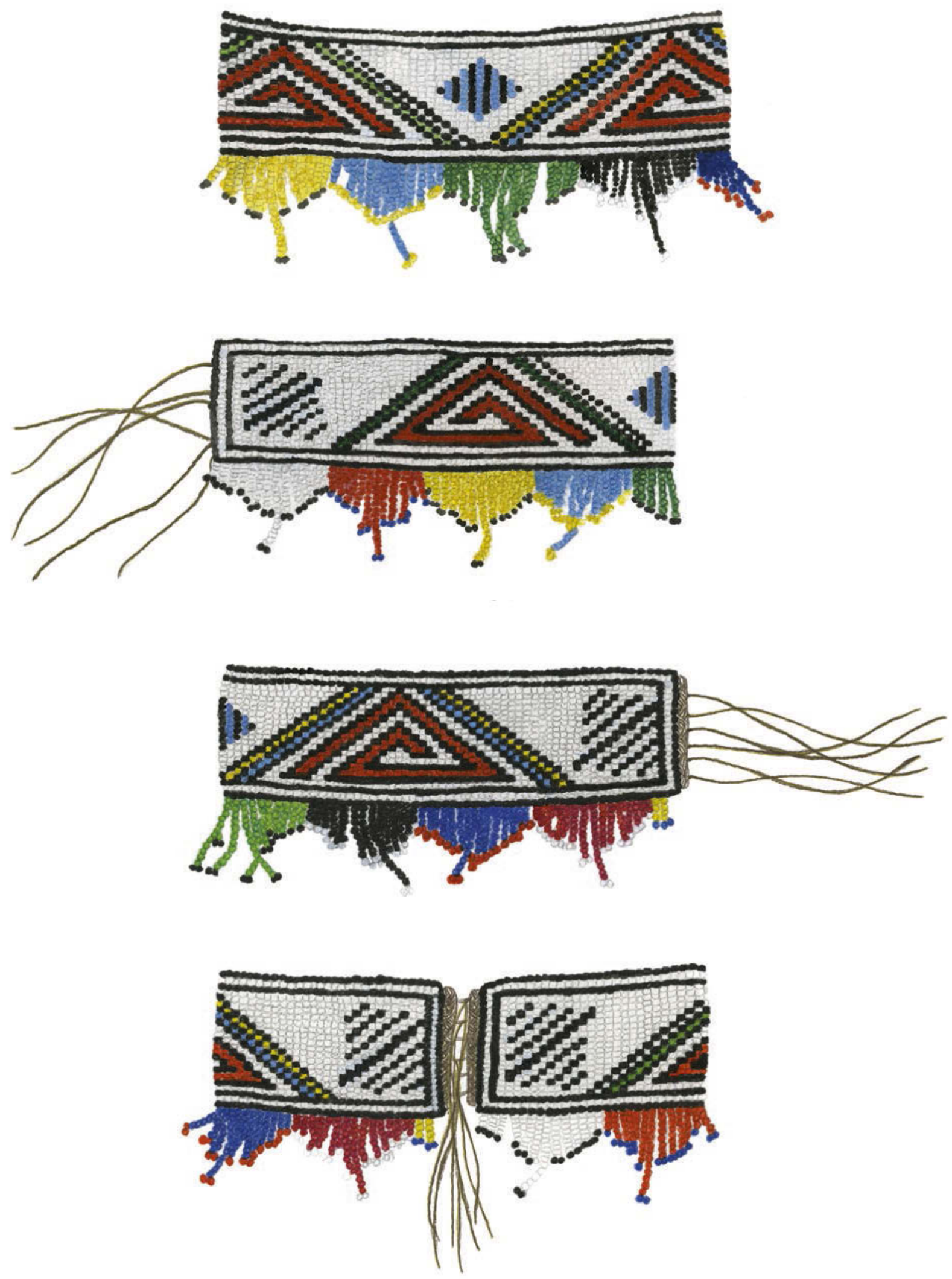

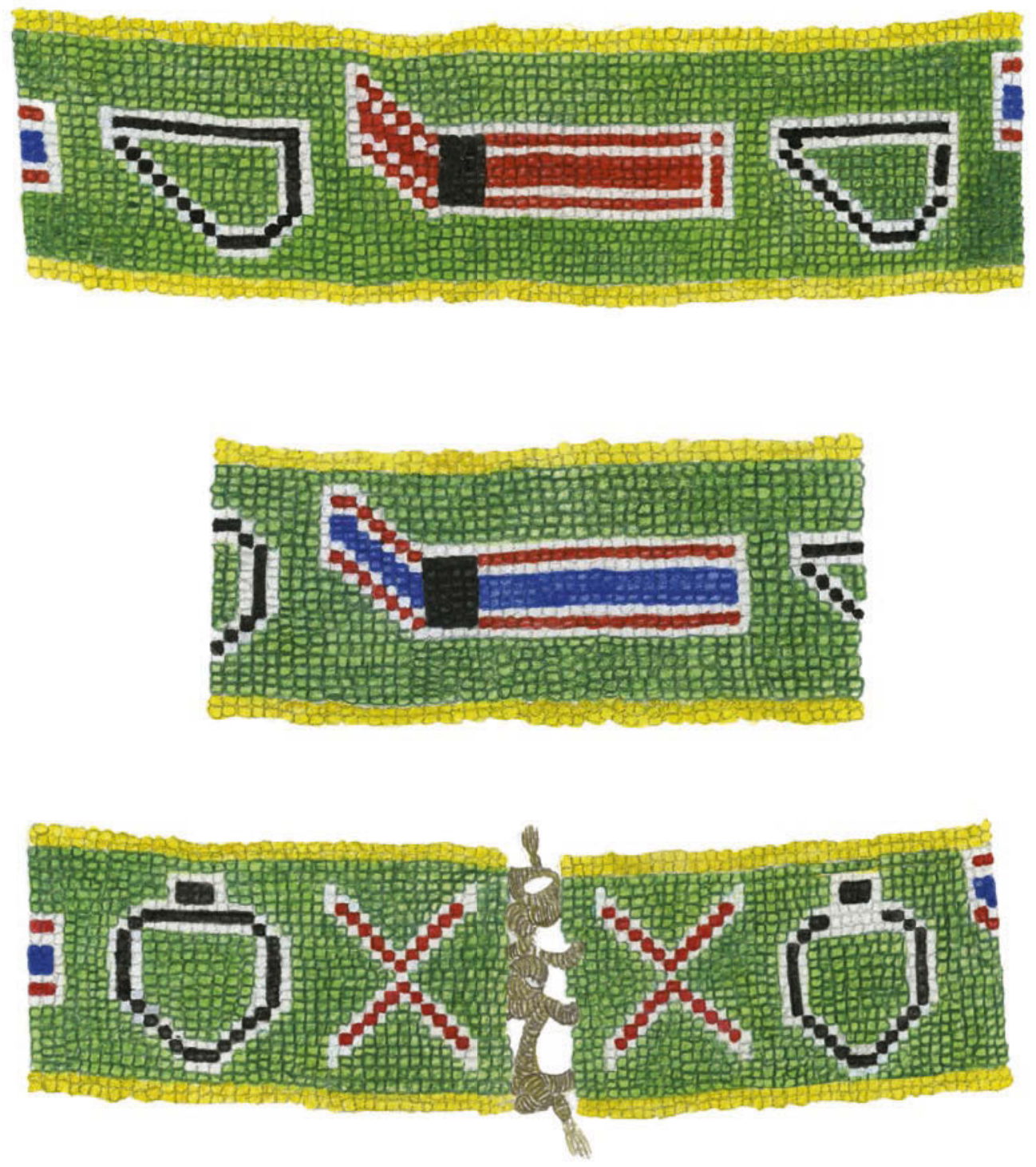
intérieur du bandeau, dont la couleur dominante est le vert de l'esprit Kapi Kene, indique que le savoir doit rester à l'intérieur d'un espace de mémorisation partagé entre des interlocuteurs.

Cette même idée d'un collectif engagé dans un processus d'apprentissage et de transmission est codée dans le bracelet utilisé par Manuel Sebastião Kanãpa (fig. 6). Celui-ci appartient à Vimini, le double proteteur de Kanãpa, désigné par la couleur blanche dominante. Aux deux extrémités, deux carrés représentent les portes de la maloca. Les deux $V$ inversés à droite et à gauche délimitent la réunion du groupe (yora anõ shoko kesoti). Quatre personnes se trouvent entre ces deux balises, figurées par les deux A orange et jaune, par le trait jaune et par la double croix orange. On m'expliqua que certains de ces personnages ont de mauvaises pensées, d'autres des bonnes. Quoi qu'il en soit, ils sont tous réunis pour parfaire leurs connaissances.

Cette alliance se renforce au rythme des divers rituels chamaniques exécutés, autant que possible, à l'intérieur des malocas. Dans ces circonstances les hommes s'assoient sur les bancs parallèles kenã, et devisent, apprennent des chants d'un maître chanteur plus âgé, décident des stratégies de cure pour soigner tel patient, reçoivent un enseignement et écoutent les chants des esprits yovevo qui viennent visiter ce monde par le truchement de chamans comme Robson. Cette image générale des rituels chamaniques est transposée dans la composition des ornements, en particulier sur le bracelet de Kanãpa. Cette image, curieusement, rappelait ou coïncidait avec notre réunion dans une salle fermée, qui avait pour objectif l'implémentation d'une autre technologie de la mémoire: le numérique.

Les motifs de base de l'iconographie (comme le triangle du motif veken kene) sont mobilisés pour construire une grammaire spécifique. Quand ils remplissent le corps au point de tracer un maillage continu de lignes de genipa ou de roucou, cette grammaire et la signification des motifs élémentaires ne sont plus pertinentes: seule compte leur concaténation qui recouvre, englobe et produit la personne afin qu'elle devienne une image reconnaissable et un interlocuteur potentiel pour les esprits. Dans ce corpus ornemental nouveau, cependant, les deux vecteurs du système visuel marubo se rencontrent: la grammaire visuelle des dessins est mobilisée pour permettre au sujet de se connecter à un «réseau d'intentionnalités complexes", selon la formule de Gell dans Art and Agency (1998). Les ornements indiquent l'existence de liens chamaniques et agissent en rendant possible la mémorisation, mais ils indiquent et agissent en disant implicitement un énoncé précis qu'on pourrait traduire de la manière suivante: «le savoir obtenu entre partenaires d'apprentissage ne doit pas être dispersé; la personne forme un cercle de confiance avec ses partenaires et ses maîtres". C'est ce qui justifie d'envisager ces ornements comme une pictographie, c'est-à-dire comme une notation graphique de parties essentielles d'un discours déterminé (Déléage 2017; Severi 2007) et en outre, ici, d'une conception générale de l'apprentissage chamanique ${ }^{13}$. Cette qualification n'épuise pas le phénomène en question, puisque cette iconographie ne saurait être analysée indépendamment de ses supports et de ses modes d'activation, sans lesquels nous manquerions sa valeur épistémologique.
13. Je tiens à remercier Emmanuel de Vienne pour m'avoir aidé à préciser ce point. 


\section{ci-contre}

fig. 6

Bracelet de Manuel

Sebastião Kanãpa en perles et fils synthétiques.

Dessin de Claudia Amara d'après les photos de Pedro de Niemeyer Cesarino.

\section{Activations technologiques}

Les explications sur le sens des ornements et sur la communauté d'apprentissage qu'ils mobilisent avaient fini par déborder sur d'autres sujets. Le double/pensée (chinã) a une «couleur », me dit Venãpa en utilisant le mot portugais (le marubo ne disposant pas d'équivalent). Les couleurs ont été établies aux temps primordiaux par Kana Voã, le principal démiurge de la mythologie marubo. C'est lui qui détermina leur répartition entre les humains et les esprits, et entre les différents «peuples» (nawavo) au sein de ces deux ensembles. Ce ne sont pas les esprits yovevo qui ont choisi le code des couleurs des ornements, mais bien Kana Voã (Isko Osho ne fait que répéter ou expliquer ce qu'il a appris du démiurge).

De la même manière que les équipes de football se distinguent par leurs couleurs, m'expliquaient-ils, les esprits sont divisés en groupes auxquels des humains peuvent finir par se relier par la parenté. Chaque groupe a son propre enseignement, comme celui qu'Isko Osho a dispensé à ses parents, une forme de transmission qu'ils comparent à l'école. Lorsque quelqu'un porte son ornement, il rêve de son protecteur/professeur; il appartiendra chaque jour un peu plus à son groupe et ne le quittera plus. La dualité asymétrique caractéristique des paires et leurs couleurs s'explique par la dualité généralisée qui caractérise le cosmos, selon Venãpa. C'est la même qui sépare la bonne et la mauvaise pensée, le Soleil et la Lune, le chemin des morts et le chemin des esprits, les deux yeux, la gauche et la droite et d'autres phénomènes.

Nous avons vu que les ornements sont utilisés seulement par ceux dont les doubles intérieurs (vaká) ont été emportés par les doubles ou les esprits qui composent la légion d'Isko Osho (les huit du tableau 3). L'ordre des unités graphiques, le design général des ornements et la distribution des couleurs sont déterminés par Isko Osho qui, à travers le corps de Robson Venãpa, enseigne aux femmes comment fabriquer les pièces. Dans les jours qui suivent, elles les tissent avec des perles achetées en ville et les rapportent à Isko Osho, qui les «souffle-chantera» (shõka, un des arts verbaux marubo) une nuit qu'il aura décidé d'apparaître sur la terre (car il s'agit d'un double poketaya, ou «voyageur», nomade). Les motifs sont donc conçus par Isko Osho, non par les femmes qui n'en sont que les exécutantes. Celles-ci livrent ensuite les ornements à Isko Osho ou à l'esprit concerné qui les pose verticalement sur son plexus solaire, donc sur celui de Robson-enveloppe. Peu après on entend un bruit, nnnnnnn, et les ornements disparaissent. C'est là un exemple supplémentaire de construction en abyme caractéristique de la multipositionalité chamanique: le plexus solaire de l'esprit est comme encastré dans le plexus solaire du chaman, qui fonctionne comme un portail ou un passage vers la dimension virtuelle du corps-maloca, d'où l'objet pourra circuler dans la dimension invisible.

Quelque temps après, le double ou l'esprit revient avec la pièce dans le corps-enveloppe de Robson. Désormais l'ornement «a déjà la clarté de l'esprit ", c'est-à-dire son chinã, m'expliquèrent-ils en portugais. La pièce ne serait donc rien si elle ne passait par ce processus d'activation. Elle ne peut dès lors être utilisée par nul autre que celui pour qui elle a été faite, ni être manipulée par quiconque aurait une «mauvaise pensée». En l'occurrence, je n'ai moi-même pu les toucher que parce que mon statut de professeur et de traducteur me valait d'être considéré comme ayant une «bonne pensée». Ils me demandèrent si je ne souhaitais pas moi aussi un ornement similaire 


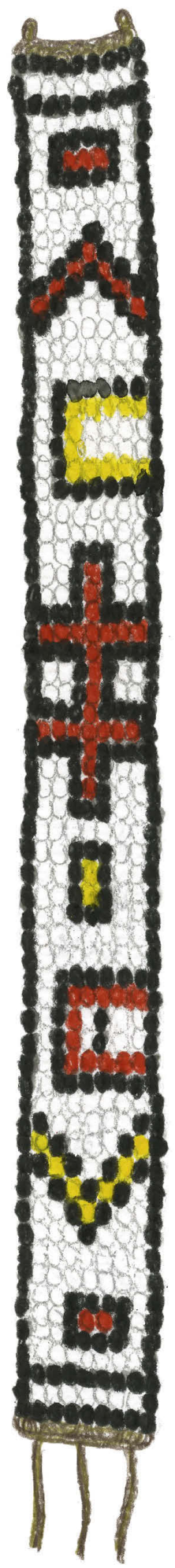


et m'invitèrent à choisir un double pour m'accompagner. Quelqu'un objecta que mon double vivait déjà dans un autre endroit, sous la garde d'une autre légion d'esprits, mais Robson Venãpa assura que cela ne posait pas de problème, car je faisais déjà partie, d'une certaine manière, de ce groupe de connaissance et de parenté. J'ai donc choisi Isko Osho et un ornement jaune, qui aurait été fabriqué si j'avais pu acheter des perles sur le moment, ce qui n’a pas été le cas.

Pour quelle raison alors les Marubo avaient-ils décidé d'utiliser cet ensemble d'ornements précisément dans le cadre de l'atelier que je leur proposais à Cruzeiro do Sul? J'y organisais, comme je l'ai indiqué, un entrâ̂nement pour la recherche, l'enregistrement et la documentation de leurs arts verbaux. Un des objectifs du transfert technologique était de formaliser le don de deux enregistreurs numériques sophistiqués, accompagnés de disques durs, et de former les Marubo à leur utilisation. Les enregistreurs devaient rester sous la responsabilité de deux personnes chargées de coordonner la poursuite du travail d'enregistrement et de documentation que j'avais mis en route. Mais les Marubo font déjà à leur manière quelque chose d'analogue à la recherche anthropologique. Leur processus d'apprentissage et de transmission du savoir chamanique, qui consiste très largement en des arts verbaux, est précisément le type d'événement qui se voit codifié par les ornements. Rien de plus naturel par conséquent que d'y avoir recours à l'occasion de l'atelier qui, de leur point de vue, était en vérité davantage un événement chamanique, puisqu'il impliquait l'utilisation d'une technologie destinée à connecter des personnes et à stocker des savoirs.

En quoi consiste, en fin de compte, le «chamanisme marubo »? Cette expression générique se réfère, en toute rigueur, à une technique d'obtention et d'enregistrement de savoirs venus d'ailleurs (des esprits yovevo, qui les délivrent aux chamans romeya), qui se distingue néanmoins radicalement de celle qui emploie des enregistreurs et l'écriture. C'est selon cette seconde méthode que j'ai travaillé durant des années, en enregistrant, annotant et recevant leurs explications à l'occasion des rituels tenus dans les malocas. L'usage des ornements indique, d'un côté, que l'accès aux technologies numériques et à l'écriture n'enclenche ni rupture ni acculturation des savoirs chamaniques, mais une transformation et une incorporation à travers des mécanismes et des catégories de pensée déjà constitués. II révèle aussi une différence profonde: l'autonomie de l'outil technologique numérique par rapport au corps, sa capacité de gérer et stocker des données indépendamment et en dépit de son opérateur, quand bien même celui-ci appuie sur les commandes, oriente les micros, s'y apparie tel un cyborg, etc. Pourquoi cette autonomie serait-elle donc nécessaire, quand bien même les instruments sophistiqués comme les ordinateurs, les caméras et les magnétophones sont effectivement désirés par les Marubo et tant d'autres sociétés amazoniennes? Ainsi, même quand ils les acquièrent, dans quelle mesure leur usage devrait-il produire chez eux un couplage cyborg aussi effectif que celui qui caractérise chaque jour un peu plus notre rapport digitalisé et prothétique avec le savoir et ses réseaux virtuels de mémoire? Leur intérêt pour nos supports technologiques ne cacherait-il pas un rapprochement comparatiste avec une autre technologie, déjà connue et maîtrisée depuis longtemps: la maîtrise du virtuel par la récursivité et la multipositionalité dont sont capables les corps chamaniques? 
Dans son étude récente sur les Araweté, Guilherme Heurich (2015: 55ss) montre que leur intérêt pour les clés USB a crû de manière exponentielle depuis le début de sa recherche tournée, comme la mienne, vers l'enregistrement et la documentation de chants. Les Arawété demandent désormais sans relâche à écouter le "pajé dans la clé USB " et passent eux-mêmes documents et enregistrements d'un support à l'autre. L'usage de ces outils n'a pas, selon lui, remis en cause la centralité des performances chamaniques, chargées des relations entre les Arawété et une myriade d'agents invisibles. Lorsque je vivais dans les villages marubo, j'employais une technologie moins sophistiquée: cassettes audio et ensuite mini-discs, bien plus chers et difficiles d'accès que les clés USB. J'ai noté rapidement l'acquisition et l'utilisation intense des magnétophones, dont les cassettes ont commencé à circuler entre les chanteurs. Si les plus expérimentés ne voulaient pas autre chose qu'écouter et rire d'entendre leur propre voix, les apprentis espéraient que cette technologie excorporée serait capable de transformer une fois pour toutes l'apprentissage de ces chants longs et complexes. Erreur, disaient Robson et bien d'autres pajés, car le savoir n'existe qu'effectivement disposé dans la chair (nami). Habitué à recourir à d'autres formes de savoir (l'écriture alphabétique étant la première et la plus importante d'entre elles), Robson doit pourtant depuis longtemps négocier avec ses doubles et ses esprits auxiliaires, toujours méfiants envers ces instruments capables d'atrophier le savoir et leurs réseaux de relation. En fin de compte, le savoir sur des gens autres (ce qui définit le chamanisme) ne s'acquiert que par des visites et des voyages constants...

Les cyborgs mélanésiens, écrivait Marilyn Strathern dans Partial Connections, sont autre chose que les "engins mi-humains, mi-mécaniques" de Donna Haraway, car ils sont "conceptuellement "taillés" dans un même matériau». L'auteur explique, en d'autres termes, qu'il n'y a pas «de différence entre un collier de coquillages et un matrilignage, entre un homme et un poteau de bambou, entre un yam et un esprit. L'un "est" l'autre dans la mesure où ils évoquent également la perception de relations " (Strathern 2005: 118). On ne saurait non plus parler de cyborgs marubo, car le présupposé d'une séparation entre un corps naturel et ses ornements culturels ou artificiels ne tient pas: tout corps pleinement humain est nécessairement un ornement ou le produit d'un procès esthétique, le corps orné est nécessairement celui qui établit des connexions avec d'autres corps virtualisés. Si l'on tenait néanmoins à sauver cette notion d'un cyborg marubo, il faudrait au moins souligner une distinction. La production d'une complexité qui déborde les limites de la peau et de l'individu et qui le transforme en nœud dans des circuits virtuels de relations ne résulte évidemment pas, comme dans le cas de Haraway, d'une révision de dualités ontologiques en place (nature/culture, masculin/féminin, organique/inorganique, etc.), pas plus que de stratégies de territorialisation et de contrôle des fluxs prévus par le capitalisme. En Amazonie, c'est le savoir dérivé des rencontres en face-à-face qui est privilégié, car un corps correctement équipé de son appareillage relationnel n'existera que s'il est composé d'autres corps qu'il s'annexe et s'il parvient, de manière analogue à la Mélanésie de Strathern, à montrer le résultat de cette quête versatile et accumulatrice du savoir.

Robson est un connecteur des temps et des positions parallèles d'où dérive son savoir. II n'est pas constructeur de la réalité qui devient visible ou audible lors des séances chamaniques où les esprits visitent la terre par son 


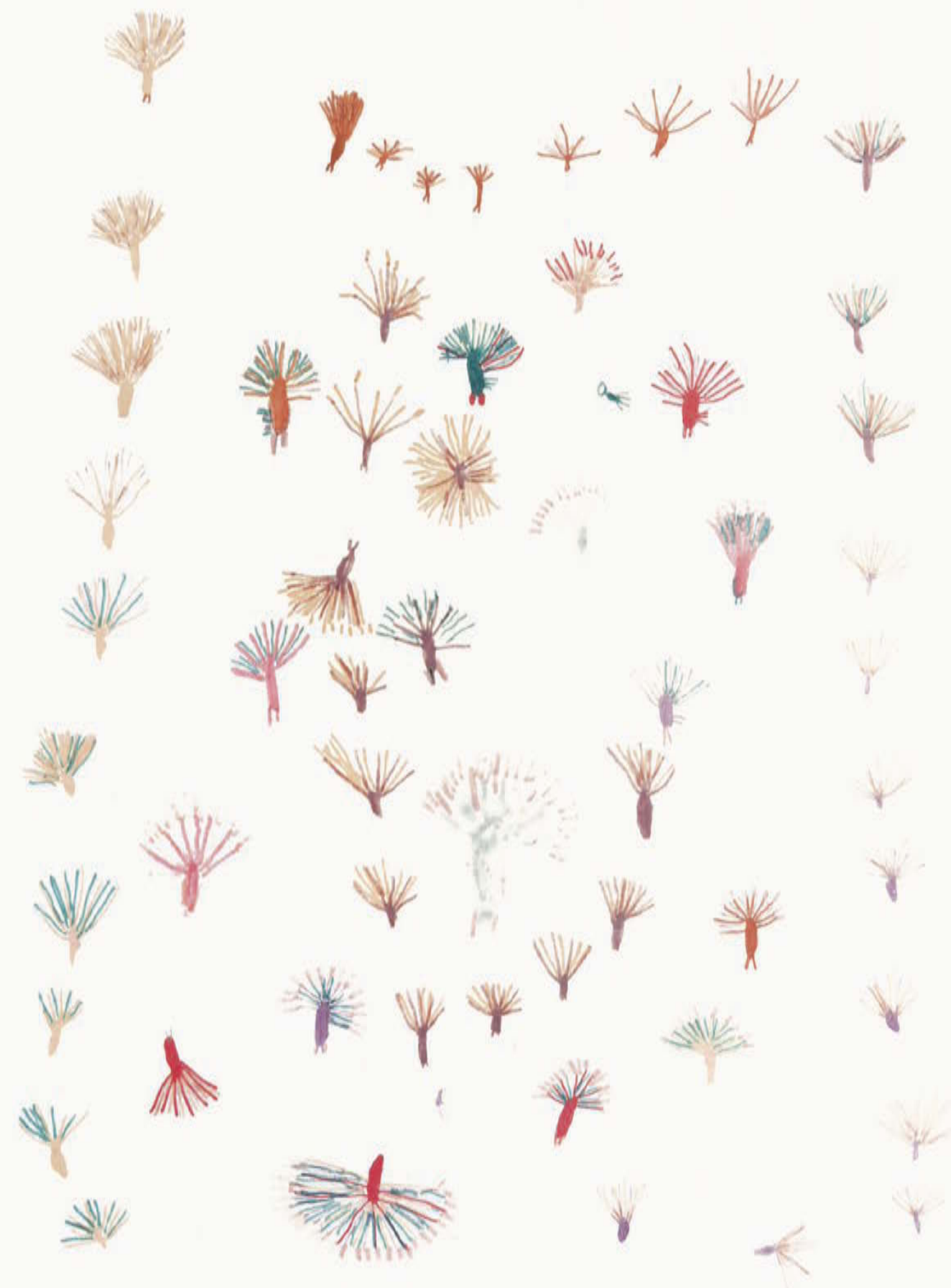




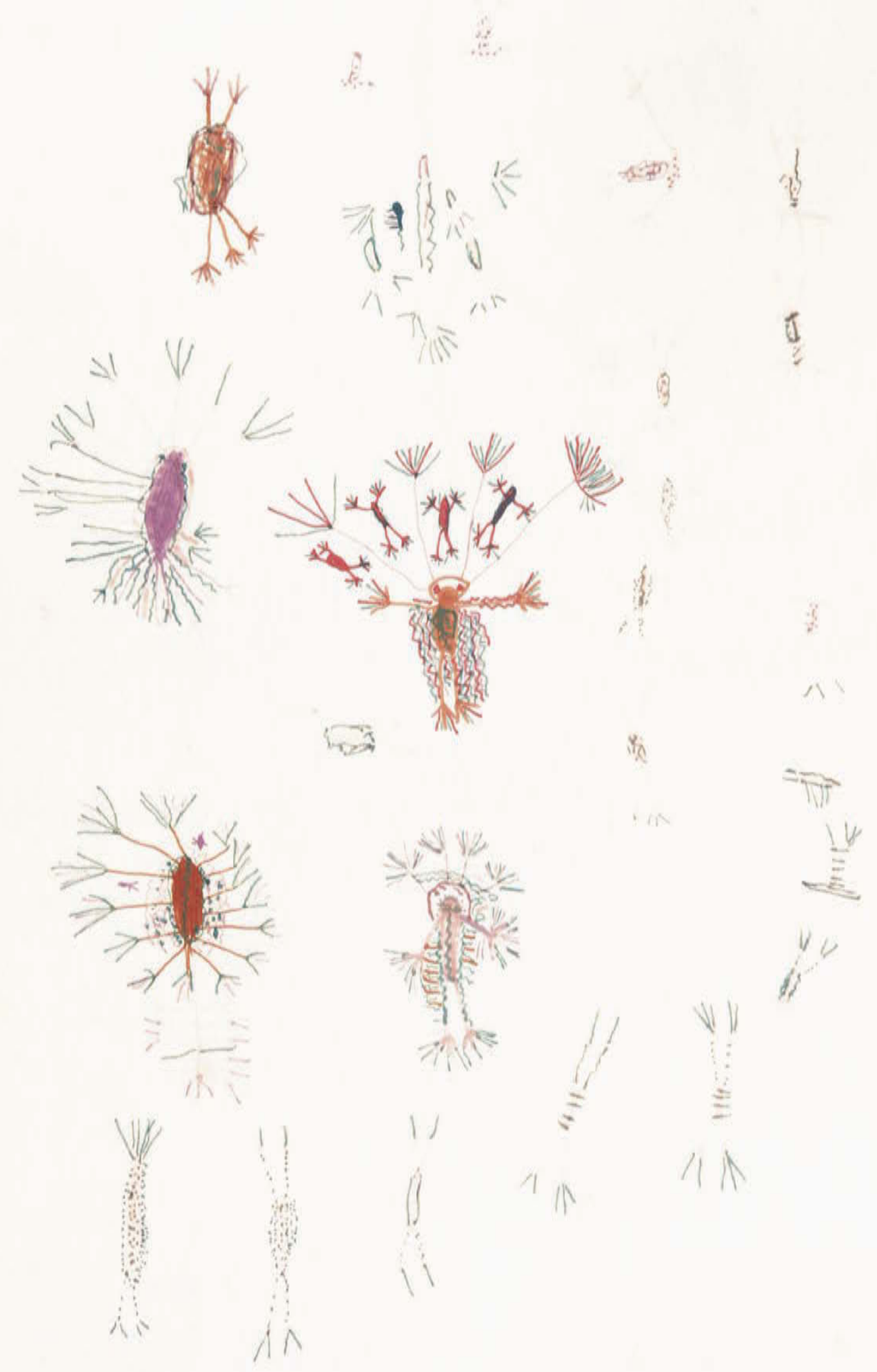


14. La notion de virtuel a été utilisée par plusieurs anthropologues, notamment Claude Lévi-Strauss et Eduardo Viveiros de Castro, pour désigner respectivement I'horizon des transformations narratives de la pensée mythique et les caractéristiques intensives des temps primordiaux. Le concept m'apparaît comme étant le plus adapté pour décrire un monde marqué non par la supposition que le sujet peut délirer ou faire des projections animiques sur le monde extérieur, mais bien plutôt par le postulat ontologique qu'il existe des positions temporelles et spatiales différentes de celles ordinairement associées à un «humain ". Le virtuel, au sens de Manuel de Landa, est auss une composante du réel il est plus précisément une "composante vitale du monde objectif" (2002: 24) et non une fiction imaginative. Le concept semble adéquat, par conséquent, pour traduire les complexes topologies chamaniques et leurs relations au savoir. Voir Cesarino 2018 pour des développements sur ce sujet.

\section{double page}

précédente

fig. 7

À gauche: Orlando Nakeuxima, L'homme (paruri) qui symbolise la nuit (titi), dessin yanomami, s.d. Collection Laymert Garcia dos Santos et Stella Senra.

fig. 8

À droite: Orlando Nakeuxima, La nuit (titi), dessin yanomami, s.d. Collection Laymert Garcia dos Santos et Stella Senra. corps. II n'est pas ainsi créateur d'une réalité, mais médiateur de temporalités et de réalités multiples qui coïncident dans sa personne et qui finissent par produire des alliances dont dérive le savoir chamanique. La tâche de «relier la pensée » (chinã ãtinãnãi), expression par laquelle les chamans marubo désignent les réseaux d'alliance établie entre humains (Cesarino 2011a), est rendue possible par la prise en charge de cette multiplicité temporelle et positionnelle par le corps du chaman (un trait qu'on retrouve dans d'autres chamanismes amazoniens, tel celui des Yanomami [Albert et Kopenawa 2010: 530]). La convergence des temporalités multiples grâce à une réalité de référence (celle, par exemple, dans laquelle nous voyons d'autres êtres transiter par le corps de Robson) peut ainsi être considérée comme un effet de position, qui n'est pas un effet d'illusion. II ne s'agit pas, comme dans certaines œuvres de Philip K. Dick, de douter du réel en y décelant la possibilité d'une prestidigitation, mais d'y postuler la virtualité d'une dérivation ${ }^{\mathbf{1 4}}$. La singularité «Robson» est toujours, ainsi, une sorte de scène du monde d'un autre (Isko Osho, Panã, etc.) qui, en se manifestant en acte, indique la potentialité d'autres mondes parallèles qui sont néanmoins assez différents des mondes privés de Dick. Du point de vue épistémologique et ontologique, ces positions ne sont en effet pas propres à Robson, puisque d'autres y participent: des chamans romeya marubo mais aussi l'«intériorité» (c'est-à-dire le corps-maison) de personnes ordinaires qui sont alliées de sa «position» (moi, entre autres) et sont visitées par des doubles et des esprits auxiliaires.

La technologie corporelle chamanique n'est pas celle d'une science future comme dans la science-fiction, pas plus qu'elle n'est la survivance d'une ritualisation archaïque ou primitive. La maîtrise de la réalité engagée dans le chamanisme réside dans sa capacité à médiatiser et traduire (Carneiro da Cunha 1998) les temporalités possibles projetées par les doubles et les esprits alliés, qui peuvent coïncider ou non avec le temps biographique du corps-maison du chaman (Cesarino 2011a; 2014), cette sorte de hub du trafic cosmologique. Ma technologie numérique, de son côté, était fondée sur un autre présupposé en matière de temporalité: préserver un savoir «traditionnel» (métaphore du passé) conçu comme patrimoine destiné à un «futur» collectif indéfini. La mésentente résidait dans l'idée qu'une conception linéaire de la temporalité pût coïncider avec ou être comprise par l'autre. Pourquoi insister sur la stabilisation des savoirs dans une base de données numériques alors que leurs détenteurs et énonciateurs sont disponibles et disposés à les mobiliser dans une opération de médiation chamanique? C'est là sans doute ce qui explique le désintérêt relatif et l'incompréhension des Marubo face au processus de numérisation des chants et des récits.

Nous avons vu que les ornements mettent en œuvre une sorte de médiation esthétique, capable d'indiquer que le processus chamanique d'acquisition des savoirs (permis par la production esthétique des personnes) est complémentaire (mais diamétralement opposé) à celui que proposent les Blancs. Cette médiation nouvelle est d'autant plus intéressante qu'elle fait écho aux transformations de la transmission qu'implique l'entreprise de documentation des savoirs traditionnels. II n'est pas anodin que les participants à l'atelier aient été munis de leurs propres technologies de production et de circulation des savoirs. L'atelier révèle une intersection entre équipements et procès technologiques distincts, chacun animé par la volonté de s'atteler aux transformations du chamanisme et de son épistémologie. 
Les transformations induites par l'alliance avec les Blancs (moi en l'occurrence) et leur régime de circulation des savoirs (publications, documentation) à l'œuvre dans la recherche académique et les technologies numériques ne concernent pas les mécanismes fondamentaux du chamanisme. Les chamanismes amazoniens se sont toujours constitués à partir de réseaux d'alliance et c'est, je pense, la raison pour laquelle le groupe d'Isko Osho est arrivé décoré dans un contexte qui représente justement une augmentation et une implémentation de leur réseau. L'activation de celui-ci, plus que les contenus transmis, est ce qui caractérise le mécanisme chamanique; son modus operandi est ainsi de connecter des corps et des personnes, et il ne saurait être substitué par des outils qui transforment la présence vocale et visuelle en fantasmagories numériques.

Dans les années qui ont suivi cet atelier, mes contacts avec les Marubo se sont faits plus rares. J'ai eu l'occasion de rencontrer à nouveau Robson et Reinaldo à São Paulo, curieusement pour les aider à terminer le montage d'un documentaire qu'ils avaient fait sur leurs communautés. J'ai posé des questions sur le travail qu'ils auraient dû poursuivre seuls dans leurs villages, et j'ai eu confirmation de ce que je soupçonnais déjà. Les deux équipements donnés aux Marubo sont passés de main en main, ont été perdus ou simplement cassés. Je sais qu'ils ont commencé à enregistrer, puisque ces enregistrements ont été perdus faute d'intérêt et d'organisation nécessaire pour ce type de technique et de monumentalisation de la «culture» réifiée (Carneiro da Cunha 2009). Rien de comparable à la prolifération de cinéastes, de bases de données géantes, à l'installation de centres d'assistance et de recherches collaboratives réussies comme celles qu'ont développées, par exemple, les anthropologues Carlos Fausto et Bruna Franchetto dans le Haut Xingu. À l'époque, Robson, à travers Isko Osho, était très occupé à restaurer l'ancien chemin des morts pour y recevoir un flux plus important de défunts «mal morts ", qui s'accumulent sur terre et dispersent une infinité de maladies. Nous pouvons supposer qu'il a manqué de temps pour superviser le projet de documentation. Lauro, autre chaman aujourd'hui disparu, m'a dit un jour qu'il pourrait emporter mon appareil photo afin que son double puisse rapporter des images de ses visites chez les autres êtres. J'aurais pu ainsi avoir une petite idée de ce que mes sens encore embrouillés sont incapables de percevoir. 


\section{Albert, Bruce et Kopenawa, Davi}

2010 La Chute du ciel: paroles d'um chaman yanomami.

Paris, Plon.

\section{Barcelos Neto, Aristóteles}

2008 Apapaatai: rituais de máscara no Xingu. São Paulo, Edusp.

\section{Boas, Franz}

1927 Primitive Art. Institute for Comparative Culture Research, Oslo.

\section{Carneiro da Cunha, Manuela}

1998 "Pontos de vista sobre a floresta amazônica: xamanismo e tradução ", Mana 4 (1) : 7-22

2009 Cultura com aspas. São Paulo, Cosac Naify.

\section{Cesarino,}

\section{Pedro de Niemeyer}

2011a Oniska: poética do xamanismo na Amazônia. São Paulo, Perspectiva/FAPESP.

2011b «Entre la parole et l'image: le système mythopoétique marubo", Journal de la Société des Américanistes 97 (1): 223-257.

2012 "A escrita e os corpos desenhados: transformações do conhecimento xamanístico entre os Marubo ", Revista de Antropologia 55 (1) : 75-95.

2014 "Multiple Biographies: Shamanism and Personhood Among the Marubo of Western Amazonia ", in Suzanne Oakdale et Magnus Course (dir.), Fluent Selves: Autobiography, Person and History in Lowland South America. Lincoln/ Londres, University of Nebraska Press: 121-144

2015 "Cartographies du cosmos image, parole et savoir chez les Marubo ", in Carlo Severi et Carlos Fausto (dir.), Paroles en images : écritures, corps et mémoires. Paris/Rio de Janeiro, Open Edition Press: 107-135.

2018a «Wenía - o surgimento dos antepassados ", Estudos de literatura brasileira contemporânea 58: 45-99. 2018b "Virtualidade e equivocidade do ser nos xamanismos ameríndios ", Revist do Instituto de Estudos Brasileiros 69: 267-288

\section{Cesarino, Pedro de Niemeyer et Carneiro da Cunha, Manuela (dir.)}

2014 Políticas culturais e povos indígenas. São Paulo, Cultura Acadêmica.

\section{Déléage, Pierre}

2007 "Les répertoires graphiques amazoniens", Journal de la Société des américanistes 93 (1) : 97-126.

2017 Lettres mortes. Paris, Fayard.

\section{Gell, Alfred}

1998 Art and Agency.

Oxford, Clarendon Press.

\section{Gow, Peter}

1999 "Piro Designs: Paintings as Meaningful Action in an Amazonian Lived World ", Journal of the Royal Anthropological Institute 5 (2) 229-246.

\section{Heurich, Guilherme} Orlandini

2015 «Música, morte e

esquecimento na arte verba araweté ". Thèse de doctorat, Universidade Federal do Rio de Janeiro.

\section{Landa (de), Manuel}

2002 Intensive Science and Virtual Philosophy. Londres, Bloomsbury.

\section{Lévi-Strauss, Claude}

1991 Histoire de lynx. Paris, Plon.

\section{Melatti, Julio Cezar}

1977 «Estrutura social Marubo: um sistema australiano na Amazônia ", Anuário Antropológico 76: 83-120.

\section{Mitchell, William John Thomas}

1994 Picture Theory: Essays on Verbal and Visual Representation. Chicago, The University of Chicago Press.

\section{Severi, Carlo}

2007 Le Principe de la chimère: une anthropologie de la mémoire. Paris, Rue d'Ulm/Musée du Quai Branly.

\section{Strathern, Marilyn}

2005 Partial Connections.

New York, Altamira Press.

\section{Taylor, Anne-Christine}

2003 "Les masques de la mémoire : essai sur la fonction des peintures corporelles jivaro ", L'Homme 165: 223-248. page 162 et ci-contre Orlando Nakeuxima, Motoka et ses fils, dessin yanomami, s.d. (détail). Collection Laymert Garcia dos Santos et Stella Senra. 
